\title{
Determination of High Strain-Rate Compressive Stress-Strain Loops of Selected Polymers
}

\author{
T. Yokoyama ${ }^{1, a}$ and K. Nakai ${ }^{1, b}$ \\ ${ }^{1}$ Department of Mechanical Engineering, Okayama University of Science, Okayama 700-0005, Japan \\ a yokoyama@mech.ous.ac.jp, ${ }^{b}$ nakai@mech.ous.ac.jp
}

Keywords: Dynamic energy absorption, Hopkinson bar, Polymer, Strain rate, Stress-strain loop

\begin{abstract}
Compressive stress-strain loops of selected polymers at strain rates up to nearly 800/s are determined in a strain range of nearly $8 \%$ on the standard split Hopkinson pressure bar. Four different commercially available extruded polymers are tested at room temperature. The compressive stress-strain loops at low and intermediate strain rates are measured on an Instron testing machine. The effects of strain rate on the Young's modulus, flow stress and dissipation energy are discussed. It is shown that the area included within the stress-strain loop increases with increasing strain rate as well as a given strain, that is, all four extruded polymers tested exhibit intrinsic strain-rate dependent viscoelastic behavior and a high elastic aftereffect following complete unloading.
\end{abstract}

\section{Introduction}

Polymeric materials with low mechanical impedance have widely been used in automotive, aerospace and portable electronics applications for shock and vibration absorption. In order to ensure the structural integrity of these applications from the product design stage, it is needed to have a precise knowledge of the stress-strain and energy dissipation behavior of these materials under impact loading. So far, the dynamic compressive [1-3], tensile [4, 5] and torsional [6] stress-strain responses of various polymers have often been determined on the conventional [7] or modified split Hopkinson pressure bar (SHPB). However, except for Ref. [8], most of the previous studies focused only on the dynamic stress-strain behavior during loading, and did not consider that during unloading process. Consequently, the dynamic energy absorption characteristics of polymeric materials have not been rigorously understood as yet.

The purpose of the present paper is to obtain valid compressive stress-strain loops of selected polymers at strain rates up to about $800 / \mathrm{s}$ using the conventional SHPB. Four different commercially available extruded polymers or ABS (Acrylonitrile Butadiene Styrene), HDPE (High Density Polyethylene), PP (Polypropylene) and PVC (Polyvinylchloride) were tested at room temperature. Cylindrical specimens with a slenderness ratio $l / d$ (= length/diameter) of 0.5 were used in the SHPB tests, and those with $l / d=2.0$ as recommended in the ASTM Designation were used in the low and intermediate strain rate tests. The compressive stress-strain loops at low and intermediate strain rates were measured on an Instron 5500R testing machine. The influences of strain rate on the Young's modulus, flow stress at a fixed strain of 5\% and dissipation energy were examined. It is shown that the area enclosed by the stress-strain loop increases with increasing strain rate as well as strain, that is, all four polymers tested display inherent strain-rate dependent viscoelastic behavior and delayed reversible deformation following complete unloading. It is expected that the present results will lead to a better understanding of the dynamic mechanical response of polymers.

\section{Experimental Procedure}

Test Polymers and Specimen Preparation. Four different common polymers, i.e., two amorphous polymers (ABS, PVC) and two semi-crystalline polymers (HDPE, PP) were chosen for compression tests at room temperature (see, Fig.1) Cylindrical specimens were machined out of commercial extruded rods with a diameter of nearly $10 \mathrm{~mm}$ into short cylinders with a diameter of $9 \mathrm{~mm}$. The 
static specimen's length was determined to be $l / d=2.0$ in accordance with the ASTM Designation E9-89a [9] (see, Table 1). The impact specimen's length was determined so that the slenderness ratio of $l / d(d=9 \mathrm{~mm})$ value is equal to 0.5 , which corresponds to the optimum specimen geometry for metallic materials suggested by Davies and Hunter [1] (see, Table 2). All specimens of the different four polymers were tested in the as-received state.

Table 1 Geometry of static compression specimen

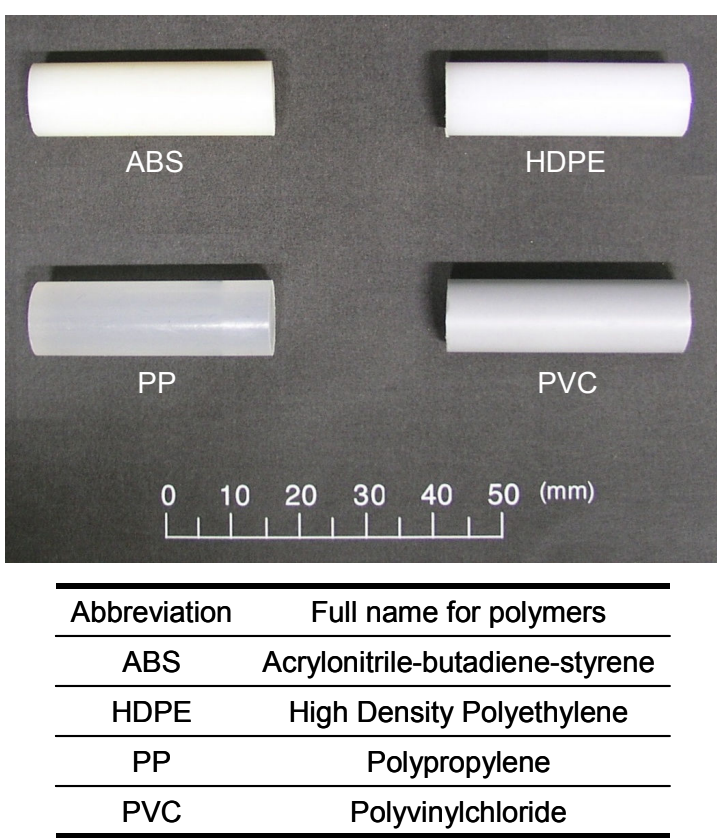

Fig.1 Picture of four different polymers tested

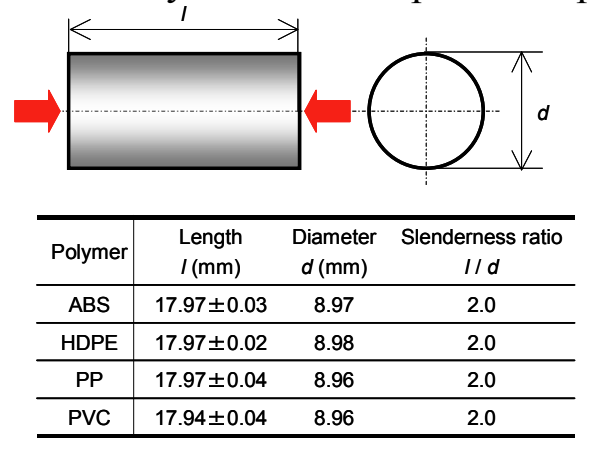

Table 2 Geometry of impact compression specimen

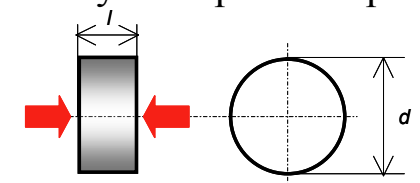

\begin{tabular}{c|ccc}
\hline Polymer & $\begin{array}{c}\text { Length } \\
I(\mathrm{~mm})\end{array}$ & $\begin{array}{c}\text { Diameter } \\
d(\mathrm{~mm})\end{array}$ & $\begin{array}{c}\text { Slenderness ratio } \\
I / d\end{array}$ \\
\hline ABS & $4.44 \pm 0.01$ & 8.98 & 0.5 \\
\hline HDPE & $4.43 \pm 0.03$ & 8.98 & 0.5 \\
\hline PP & $4.50 \pm 0.07$ & 8.97 & 0.5 \\
\hline PVC & $4.44 \pm 0.03$ & 8.97 & 0.5 \\
\hline
\end{tabular}

High Strain-Rate Compression Testing. The general arrangement of the SHPB set-up is given in Fig. 2. The SHPB set-up consists of two 2024-T4 Al alloy bars of $2000 \mathrm{~mm}$ long and $10.1 \mathrm{~mm}$ diameter, which remain elastic during the tests. A striker bar is a rod of the same material and diameter, having a length of $350 \mathrm{~mm}$. The mechanical properties of the bars are as follows: Young's modulus $E=73 \mathrm{GPa}$; longitudinal elastic wave velocity $c_{\mathrm{o}}=5130 \mathrm{~m} / \mathrm{s}$; mechanical impedance $Z=\rho$ $c_{\mathrm{o}}=14.2 \times 10^{6} \mathrm{~kg} \mathrm{~m}^{-2} \mathrm{~s}^{-1}$; yield strength $\sigma_{\mathrm{Y}}=325 \mathrm{MPa}$. The 2024-T4 Al alloy bars with low mechanical impedance are used to reduce a drastic impedance mismatch between the polymer specimen $(Z \doteqdot$ $\left.1 \sim 2 \times 10^{6} \mathrm{~kg} \mathrm{~m}^{-2} \mathrm{~s}^{-1}\right)$ and the conventional steel bars $\left(Z \doteqdot 40 \times 10^{6} \mathrm{~kg} \mathrm{~m}^{-2} \mathrm{~s}^{-1}\right)$, which results in a transmitted strain signal with a very low signal-to-noise ratio. The specimen is held in place between the input and output bars by applying a very small pre-compression load with turning of the head of a support block. As in the static tests, lubricant (petroleum jelly) is applied to the bar/specimen interfaces to minimize the frictional effects. A pulse shaping technique [8] is used to generate well-defined compressive strain pulses without inherent high-frequency noise in the input bar. Namely, a thin 1050 aluminum disk of nearly $10 \mathrm{~mm}$ diameter and $0.2 \mathrm{~mm}$ thick is attached onto the impact end of the input bar using a thin layer of petroleum jelly. When the input bar is impacted with the striker bar launched through the gun barrel, a compressive strain pulse $\left(\varepsilon_{i}\right)$ is generated in the input bar and travels towards the specimen. At the bar/specimen interface, because of the impedance mismatch, part of the strain pulse is reflected back into the input bar $\left(\varepsilon_{r}\right)$ and the remaining part is transmitted through the specimen into the output bar $\left(\varepsilon_{t}\right)$. Figure 3 gives a Lagrangian $x$ - $t$ diagram illustrating the details of the strain pulse propagation in the Hopkinson bars. Note that in the impact testing of the polymers, the duration of the reflected and transmitted strain pulses commonly becomes much longer than that of the incident strain pulse $\left(\varepsilon_{i}\right)$. This is entirely due to a very long retardation time [10] of the polymers themselves. The incident, reflected and transmitted strain pulses are then 
recorded with two pairs of strain gages (gage length $=1 \mathrm{~mm}$ ) mounted on the input and output bars. The output signals from the strain gages are fed through a bridge circuit into a 10-bit digital storage oscilloscope, where the signals are digitized and stored at a sampling time of $1 \mu \mathrm{s} /$ word. The digitized data are then transferred to a 32-bit personal computer for data processing.

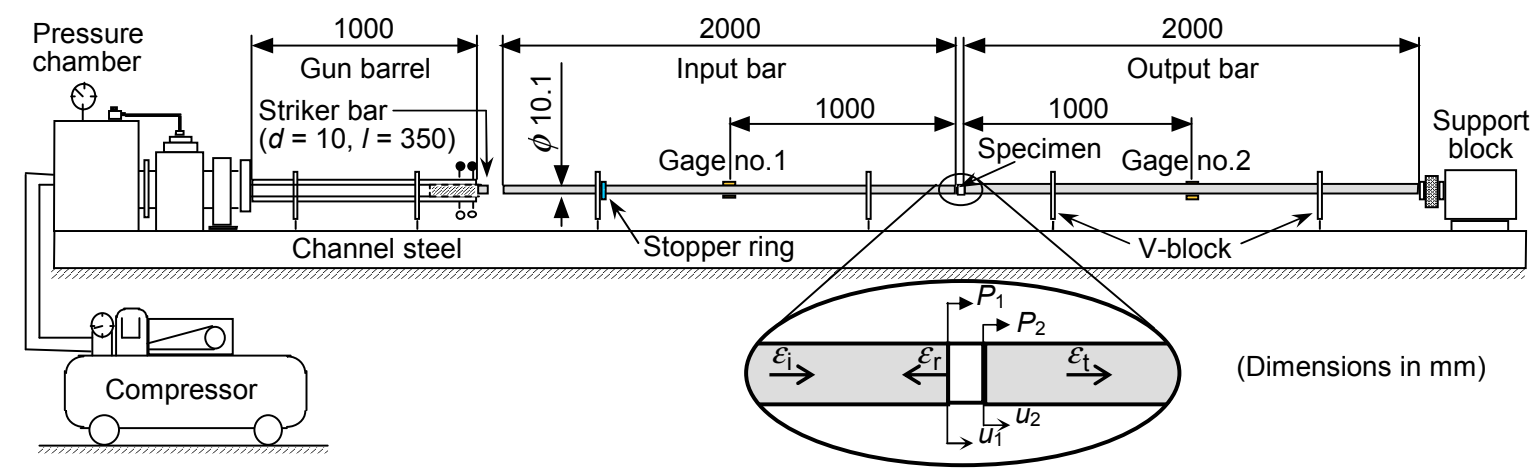

Fig.2 Schematic diagram of conventional split Hopkinson pressure bar set-up (recording system not shown)

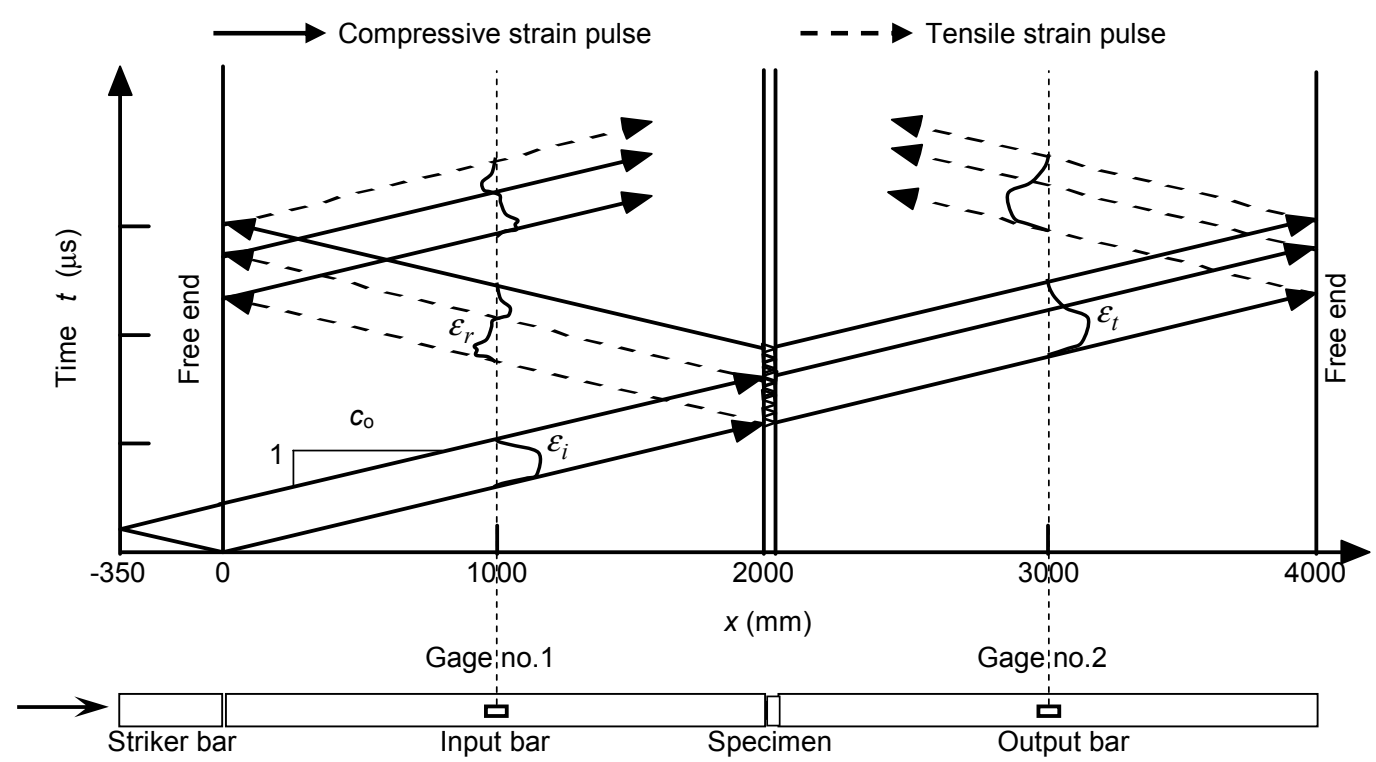

Fig.3 Lagrangian diagram for conventional split Hopkinson pressure bar

By applying the elementary one-dimensional theory of elastic wave propagation, we can determine the nominal strain $\varepsilon(t)$, strain rate $\dot{\varepsilon}(t)$ and stress $\sigma(t)$ in the specimen from the SHPB test records as [11]

$$
\begin{aligned}
& \varepsilon(t)=\frac{u_{1}(t)-u_{2}(t)}{l}=\frac{2 c_{\mathrm{o}}}{l} \int_{0}^{t}\left\{\varepsilon_{i}\left(t^{\prime}\right)-\varepsilon_{t}\left(t^{\prime}\right)\right\} \mathrm{d} t^{\prime} \\
& \dot{\varepsilon}(t)=\frac{\dot{u}_{1}(t)-\dot{u}_{2}(t)}{l}=\frac{2 c_{\mathrm{o}}}{l}\left\{\varepsilon_{i}(t)-\varepsilon_{t}(t)\right\} \\
& \sigma(t)=\frac{P_{2}(t)}{A_{\mathrm{S}}}=\frac{A E}{A_{\mathrm{S}}} \varepsilon_{t}(t)
\end{aligned}
$$


Here $u$ and $P$ are, respectively, the displacement and the axial force on both ends of the specimen (see the inset in Fig. 2). Equations (1) to (3) are derived under the assumption of dynamic force equilibrium $\left(P_{1}(t)=P_{2}(t)\right)$ across the specimen, which can be expressed as

$$
\sigma_{1}(t)=\sigma_{2}(t) \text { or } \varepsilon_{i}(t)+\varepsilon_{r}(t)=\varepsilon_{t}(t)
$$

where

$$
\sigma_{1}(t)=\frac{P_{1}(t)}{A_{\mathrm{S}}}=\frac{A E}{A_{\mathrm{S}}}\left[\varepsilon_{i}(t)+\varepsilon_{r}(t)\right], \quad \sigma(t)=\frac{P_{2}(t)}{A_{\mathrm{S}}}=\frac{A E}{A_{\mathrm{S}}} \varepsilon_{t}(t)
$$

Here $A$ and $E$ denote the cross-sectional area and Young's modulus of the Hopkinson bars; $l$ and $A_{\mathrm{S}}$ denote the length (or thickness) and cross-sectional area of the specimen. Eliminating time $t$ through Eqs. (1) to (3) yields the nominal (or engineering) compressive stress-strain and strain rate-strain relations. The compressive stress and strain are assumed to be positive in this work.

\section{Results and Discussion}

A number of the SHPB tests were conducted on the four different polymers at room temperature. Figure 4 indicates typical oscilloscope records from the SHPB test on ABS. The top trace gives the incident and reflected strain pulses $\left(\varepsilon_{i}\right.$ and $\varepsilon_{r}$ ), and the bottom trace gives the transmitted strain pulse $\left(\varepsilon_{t}\right)$. The recorded signal data are neither smoothed nor averaged electronically. It is very important to notice that the duration ( $\fallingdotseq 380 \mu$ s) of the reflected and transmitted strain pulses is much longer than that $(\fallingdotseq 270 \mu \mathrm{s})$ of the incident strain pulse. This is because it takes much time for the specimen to recover to zero stress during unloading, causing often the overlapping between the transmitted strain pulse and its strain pulse reflected from the free (or right) end of the output bar. This is graphically represented in the Lagrangian $x$ - $t$ diagram shown in Fig. 3 . In the present tests, the overlapping of the two strain pulses is successfully avoided by the use of the long output bar. Figure 5 shows the dynamic compressive stress and strain time histories for ABS. Note that the strain increases more slowly than the stress, and then decreases asymptotically to about 3.5\% strain until the stress very gradually reduces to zero at nearly $380 \mu \mathrm{s}$. Figure 6 presents the resulting dynamic stress-strain loop and strain rate-strain relations in compression. The strain rate does not remain constant during loading as well as unloading, and hence the strain rate $\dot{\varepsilon}=680$ /s given denotes the average one during loading process, which is calculated by dividing the area under the strain rate-strain curve up to the maximum strain (= $8 \%$ ) by the value of its strain. As in the low and intermediate strain rate tests, the dynamic stress-strain loop is not closed, and, consequently, a residual strain of about $3.5 \%$ is not completely recovered to zero in time.

Figures 7(a) to 7(d) show the compressive stress-strain loops of the four different polymers at three different strain rates. The intermediate strain rate stress-strain loops were measured on the Instron 5500R testing machine at a crosshead speed of $100 \mathrm{~mm} / \mathrm{min}$. It is observed that the initial slope (or Young's modulus $E$ ) and the area within the loop increase greatly with increasing strain rate. In an effort to evaluate the effects of strain rate on the overall compressive properties, the measured values for the Young's modulus, flow stress at a given strain of 5\% and dissipation energy are plotted in Figs. 8 to 10 , respectively, as functions of the average strain rate $\dot{\varepsilon}$ during loading process. Note that in the plots of the Young's modulus in Fig. 8, the strain rate in the initial elastic portion of the dynamic stress-strain loops does not exactly correspond to the average strain rate during entire loading process and, hence, the strain rate is re-evaluated by dividing the area under the initial strain rate-strain curve up to a strain at the proportional limit by the value of its strain. Thus, the average strain rate in the initial elastic region for each polymer is used in Fig.8. In Fig. 10, the dissipation energy $U_{\mathrm{d}}$ is obtained as the area enclosed by the loop, which can be calculated by subtracting the area under stress-strain curve during unloading from that during entire loading. 


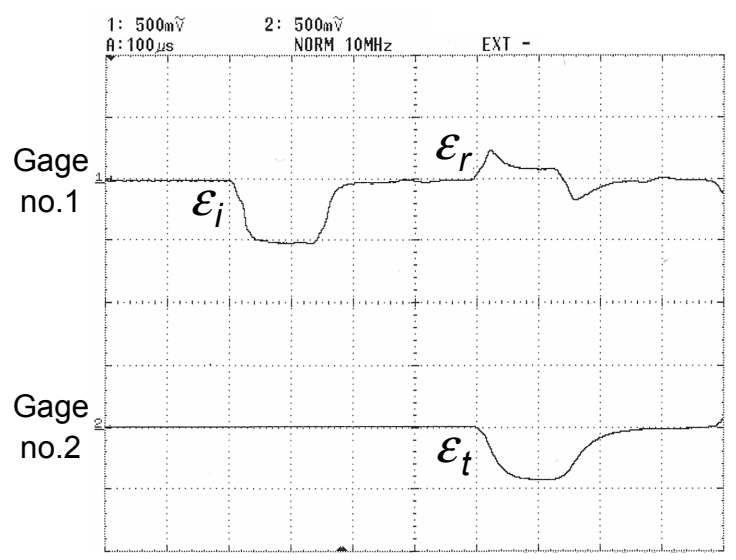

Sweep rate: $100 \mu \mathrm{s} / \mathrm{div}$

Vertical sensitivity:

Upper trace: $500 \mathrm{mV} / \mathrm{div}(1266 \mu \varepsilon / \mathrm{div})$

Lower trace: $500 \mathrm{mV} / \mathrm{div}(1274 \mu \varepsilon / \mathrm{div})$
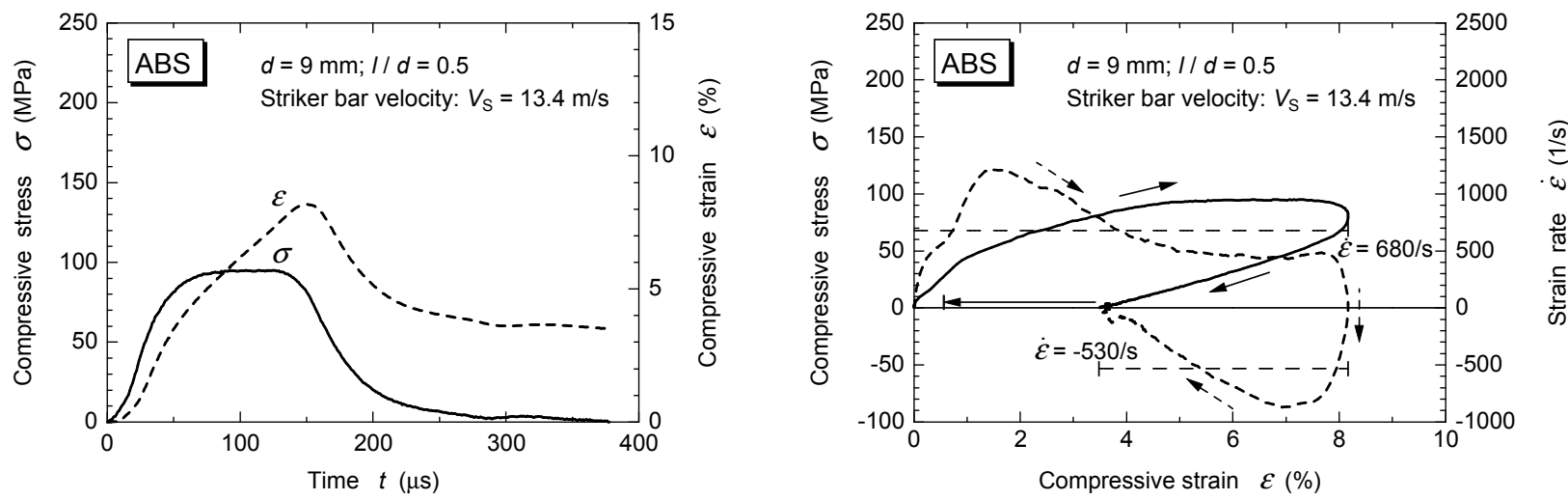

Fig. 5 Dynamic stress and strain time histories for $\mathrm{ABS}$

Fig. 6 Dynamic stress-strain and strain rate-strain loops in compression for $\mathrm{ABS}$
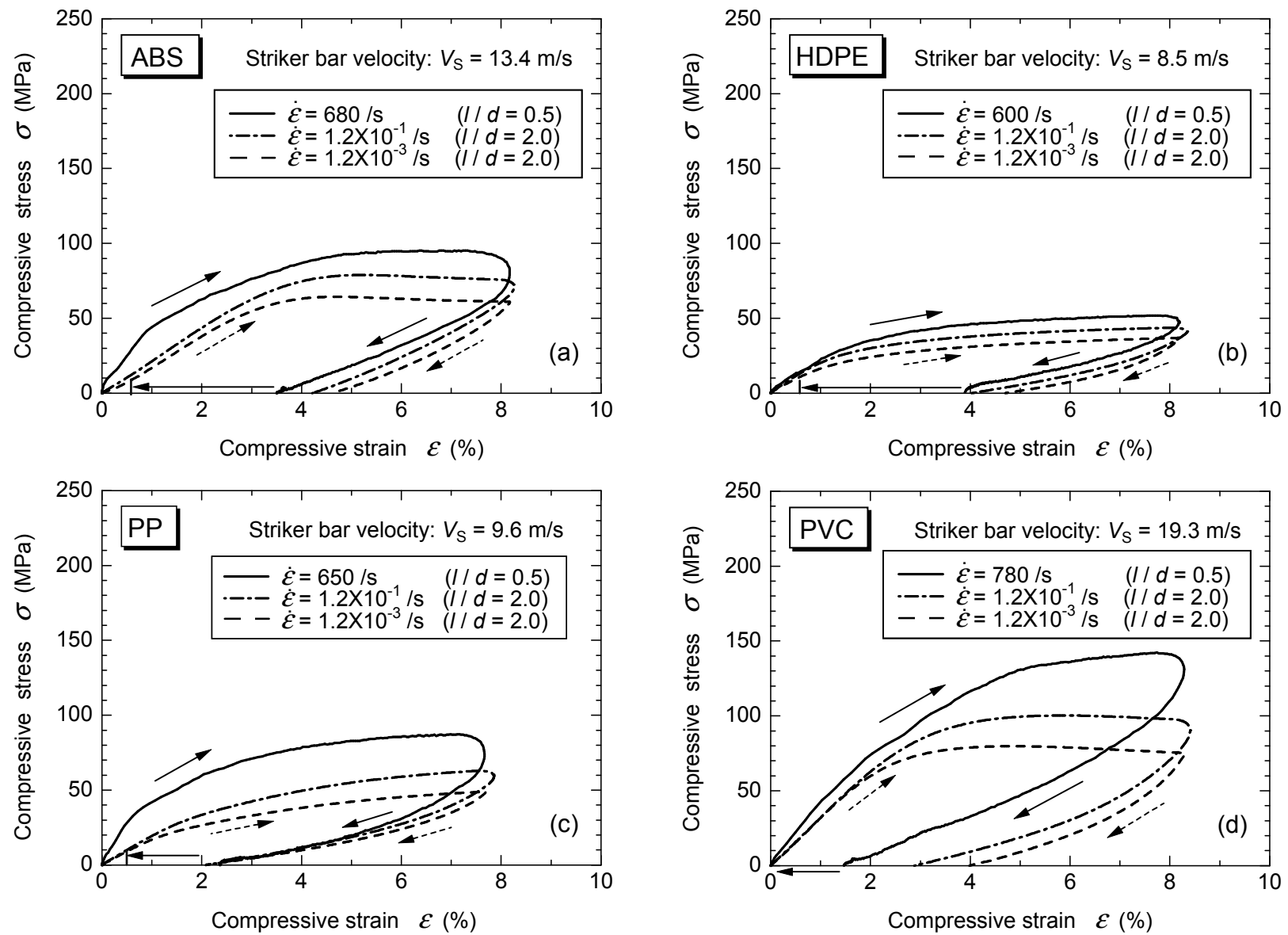

Fig. 7 Stress-strain loops in compression for four different polymers at three different strain rates 


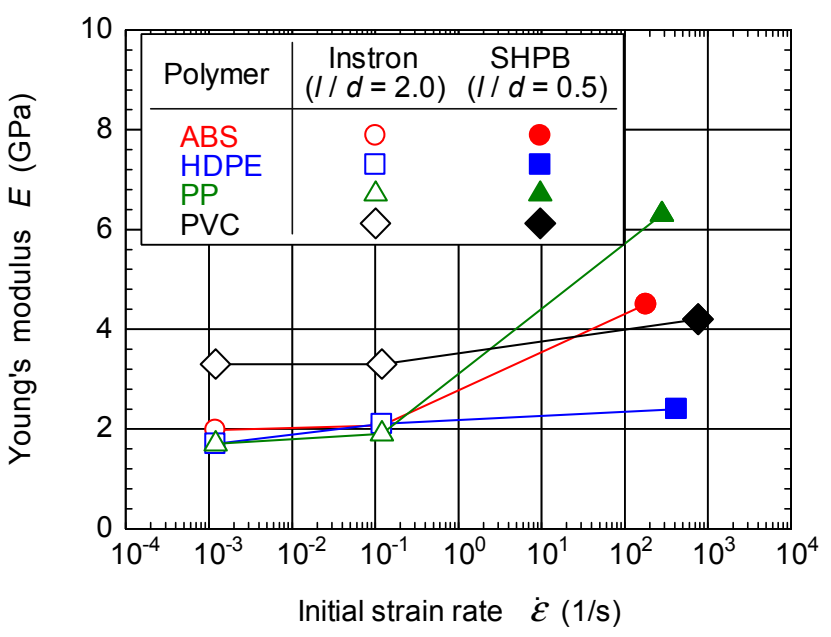

Fig.8 Young's modulus versus initial strain rate for four different polymers

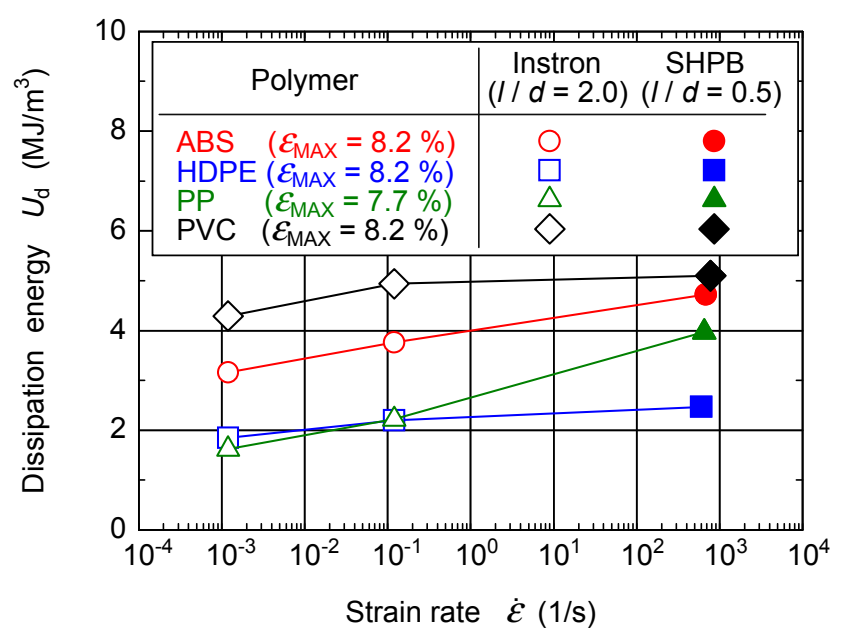

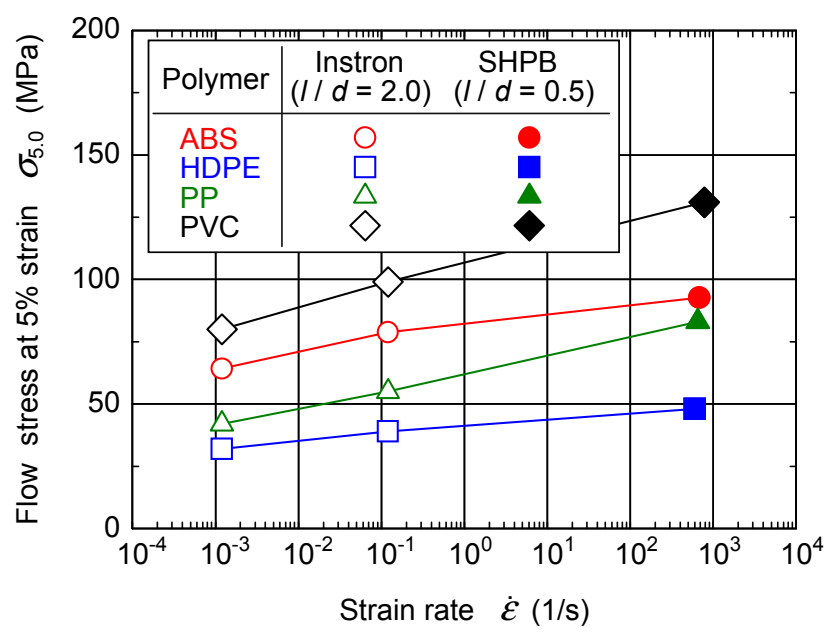

Fig.9 Flow stress at 5\% strain versus strain rate for four different polymers

Fig.10 Dissipation energy versus strain rate for four different polymers. Note: strain values in parentheses denote the maximum compressive strain achieved in each SHPB test

As can be seen from Figs. 8 to 10, the Young's modulus, flow stress at the fixed strain of 5\% and dissipation energy increase significantly with increasing strain rate. Amorphous polymers (ABS and PVC) exhibit a higher flow stress and a larger dissipation energy than semi-crystalline polymers (HDPE and PP) at any strain rate. The dissipation energy is mostly converted to heat during high rate deformation, which causes the adiabatic temperature rise within the specimen. The temperature rise $\Delta T_{\mathrm{d}}$ can be evaluated based on the dynamic dissipation energy $U_{\mathrm{d}}$ as (see Ref. [12]):

$$
\Delta T_{\mathrm{d}}=\frac{\beta_{\mathrm{C}}}{\rho c_{\mathrm{p}}} \oint \sigma(\varepsilon) \mathrm{d} \varepsilon=\frac{\beta_{\mathrm{C}}}{\rho c_{\mathrm{p}}} U_{d}
$$

Here the numerical integration is performed over $\left[0, \varepsilon_{\max }\right] ; \beta_{\mathrm{C}}(=0.9)$ denotes the conversion factor; $\rho$ and $c_{\mathrm{p}}$ are the mass density and the specific heat listed in Table $3 ; \sigma(\varepsilon)$ is the dynamic stress-strain loop. The temperature rise in each polymer calculated from Eq.(5) is given also in Table 3. Of the four polymers, the highest temperature rise is clearly observed in PVC during high rate deformation up to nearly the same strain of $8 \%$.

Table 3 Temperature rise in four polymers tested during high rate deformation

\begin{tabular}{c|cccc}
\hline & $\mathrm{ABS}(\mathbf{O})^{*}$ & $\mathrm{HDPE}(\mathbf{\square})$ & $\mathrm{PP}(\mathbf{\Delta})$ & $\mathrm{PVC}(\bullet)$ \\
\hline$\rho\left(\mathrm{kg} / \mathrm{m}^{3}\right)$ & 1040 & 960 & 900 & 1420 \\
\hline$c_{\mathrm{p}}(\mathrm{kJ} / \mathrm{kg} \cdot \mathrm{K})$ & $(1.53)^{\star \star}$ & $(2.30)$ & $(1.93)$ & $(1.01)$ \\
\hline$U_{\mathrm{d}}\left(\mathrm{MJ} / \mathrm{m}^{3}\right)$ & 4.72 & 2.47 & 3.97 & 5.10 \\
\hline$\Delta T_{\mathrm{d}}(\mathrm{K})$ & 2.67 & 1.01 & 2.05 & 3.20
\end{tabular}

* Note: symbols in parentheses correspond to those given in Fig. 10

** Note: values in parentheses are provided by manufacturers 


\section{Conclusions}

From the present experimental work, we can draw the following conclusions:

(1) All four polymers exhibit intrinsic strain-rate dependent viscoelastic behavior and a high elastic aftereffect following complete unloading.

(2) The Young's modulus, flow stress at the fixed strain of 5\% and dissipation energy for all four polymers increase greatly with increasing strain rate.

(3) The highest temperature rise is found in PVC during high strain-rate deformation up to nearly $8 \%$ strain.

(4) Amorphous polymers (ABS and PVC) have higher flow stress and larger dissipation energy than semi-crystalline polymers (HDPE and PP) at any strain rate.

\section{References}

[1] E.D.H. Davies and S.C. Hunter: J. Mech. Phys. Solids, Vol. 11 (1963), p. 155.

[2] S.S. Chiu and V.H. Neubert: J. Mech. Phys. Solids, Vol. 15 (1967), p. 177.

[3] E.W. Billington and C. Brissenden: Int. J. Mech. Sci., Vol. 13 (1971), p. 531.

[4] W. Chen, F. Lu and M. Cheng: Polymer Testing, Vol. 21 (2002), p. 113.

[5] V.P.W. Shim, J. Yuan and S.-H. Lee: Exp. Mech., Vol. 41 (2001), p. 122.

[6] N.A. Fleck, W.J. Stronge and J.H. Liu: Proc. Roy. Soc. Lon., Vol. A429 (1990), p. 459.

[7] H. Kolsky: Proc. Phys. Soc., Vol. B62 (1949), p. 676.

[8] B. Song and W. Chen: Exp. Mech., Vol. 44 (2004), p. 622.

[9] ASTM E9-89a: Annual Book of ASTM Standards. Vol. 03.01, p. 98, American Society for Testing and Materials, Philadelphia (1995).

[10] I.M. Ward and J. Sweeney: An Introduction to the Mechanical Properties of Solid Polymers, 2nd Edition, p. 57, John Wiley \& Sons, Chichester (2004).

[11] U.S. Lindholm: J. Mech. Phys. Solids, Vol. 12 (1964), p. 317.

[12] M.A.Meyers: Dynamic Behavior of Materials, p. 377, John Wiley \& Sons, London (1994). 